International Journal of Pure and Applied Mathematics

Volume 96 No. 1 2014, 37-45

ISSN: 1311-8080 (printed version); ISSN: 1314-3395 (on-line version)

url: http://www.ijpam.eu

doi: http://dx.doi.org/10.12732/ijpam.v96i1.4

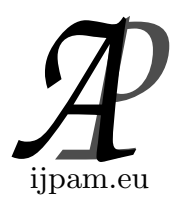

\title{
THE MONOPHONIC GRAPHOIDAL COVERING NUMBER OF A GRAPH
}

\author{
P. Titus ${ }^{1 \S}$, S. Santha Kumari ${ }^{2}$ \\ ${ }^{1}$ Department of Mathematics \\ University College of Engineering Nagercoil \\ Anna University \\ Tirunelveli Region \\ Nagercoil, 629 004, INDIA \\ ${ }^{2}$ Department of Mathematics \\ Udaya School of Engineering \\ Vellamodi, 629 204, INDIA
}

\begin{abstract}
A chord of a path $P$ is an edge joining two non-adjacent vertices of $P$. A path $P$ is called a monophonic path if it is a chordless path. A monophonic graphoidal cover of a graph $G$ is a collection $\psi_{m}$ of monophonic paths in $G$ such that every vertex of $G$ is an internal vertex of at most one monophonic path in $\psi_{m}$ and every edge of $G$ is in exactly one monophonic path in $\psi_{m}$. The minimum cardinality of a monophonic graphoidal cover of $G$ is called the monophonic graphoidal covering number of $G$ and is denoted by $\eta_{m}$. We determine bounds for it and characterize graphs which realize these bounds. Also, for any positive integer $n$ with $q-p+2 \leq n \leq q-1$, there exists a tree $T$ such that the monophonic graphoidal covering number is $n$.
\end{abstract}

AMS Subject Classification: $05 \mathrm{C} 70$

Key Words: graphoidal cover, acyclic graphoidal cover, geodesic graphoidal cover, monophonic path, monophonic graphoidal cover, monophonic graphoidal covering number

Received: $\quad$ March 21, 2014

(C) 2014 Academic Publications, Ltd. url: www.acadpubl.eu

${ }^{\S}$ Correspondence author 


\section{Introduction}

By a graph $G=(V, E)$ we mean a finite, undirected connected graph without loops or multiple edges. The order and size of $G$ are denoted by $p$ and $q$ respectively. For basic graph theoretic terminology we refer to Harary[6]. The concept of graphoidal cover was introduced by Acharya and Sampathkumar[2] and further studied in $[1,3,7,8]$.

A graphoidal cover of a graph $G$ is a collection $\psi$ of (not necessarily open) paths in $G$ satisfying the following conditions.

(i) Every path in $\psi$ has at least two vertices.

(ii) Every vertex of $G$ is an internal vertex of at most one path in $\psi$.

(iii) Every edge of $G$ is in exactly one path in $\psi$.

The minimum cardinality of a graphoidal cover of $G$ is called the graphoidal covering number of $G$ and is denoted by $\eta(G)$.

The collection $\psi$ is called an acyclic graphoidal cover of $G$ if no member of $\psi$ is cycle; it is called a geodesic graphoidal cover if every member of $\psi$ is a shortest path in $G$. The minimum cardinality of an acyclic (geodesic) graphoidal cover of $G$ is called the acyclic (geodesic) graphoidal covering number of $G$ and is denoted by $\eta_{a}\left(\eta_{g}\right)$. The acyclic graphoidal covering number and geodesic graphoidal covering number are studied in $[4,5]$.

A chord of a path $P$ is an edge joining any two non-adjacent vertices of $P$. A path $P$ is called a monophonic path if it is a chordless path. For any two vertices $u$ and $v$ in a connected graph $G$, the monophonic distance $d_{m}(u, v)$ from $u$ to $v$ is defined as the length of a longest $u-v$ monophonic path in $G$. The monophonic eccentricity $e_{m}(v)$ of a vertex $v$ in $G$ is $e_{m}(v)=\max \left\{d_{m}(v, u): u \in V(G)\right\}$. The monophonic radius is $\operatorname{rad}_{m}(G)=\min \left\{e_{m}(v): v \in V(G)\right\}$ and the monophonic diameter is $\operatorname{diam}_{m}(G)=\max \left\{e_{m}(v): v \in V(G)\right\}$. The monophonic distance was introduced and studied in $[9,10]$.

The following theorems will be used in the sequal.

Theorem 1.1. [6] Every non-trivial connected graph has at least two vertices which are not cut vertices.

Theorem 1.2. [6] Let $G$ be a connected graph with at least three vertices. The following statements are equivalent:

(i) $G$ is a block

(ii) Every two vertices of $G$ lie on a common cycle.

Theorem 1.3. [5] Let $K_{m, n}(1 \leq m \leq n)$ be a bipartite graph. Then 


$$
\eta_{g}\left(K_{m, n}\right)= \begin{cases}1 & \text { if } m=1, n=1 \\ n-1 & \text { if } m=1, n \geq 2 \\ n & \text { if } m=2, n \geq 2 \\ m+n-1 & \text { if } m=3, n=3,4 \\ m+n & \text { if } m=3, n=5 \\ 2 n-3 & \text { if } m=3, n \geq 6 \\ m n-m-n & \text { if } m, n \geq 4 .\end{cases}
$$

Throughout this paper $G$ denotes a connected graph with at least two vertices.

\section{Monophonic Graphoidal Cover}

Definition 2.1. A monophonic graphoidal cover of a graph $G$ is a collection $\psi_{m}$ of monophonic paths in $G$ such that every vertex of $G$ is an internal vertex of at most one monophonic path in $\psi_{m}$ and every edge of $G$ is in exactly one monophonic path in $\psi_{m}$. The minimum cardinality of a monophonic graphoidal cover of $G$ is called the monophonic graphoidal covering number of $G$ and is denoted by $\eta_{m}(G)$.

Example 2.2. For the graph $G$ given in Figure 2.1, $\psi_{m}=\left\{\left(v_{1}, v_{2}, v_{3}, v_{4}, v_{5}, v_{6}, v_{7}\right),\left(v_{3}, v_{10}, v_{1}, v_{8}, v_{7}, v_{9}, v_{5}\right)\right\}$ is a minimum monophonic graphoidal cover of $G$ and so $\eta_{m}(G)=2$.

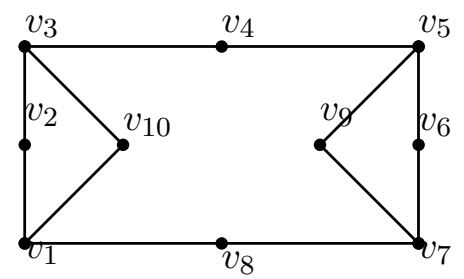

Figure 2.1: $G$

Theorem 2.3. $\quad$ For any connected graph $G, \eta(G) \leq \eta_{a}(G) \leq \eta_{m}(G)$ $\leq \eta_{g}(G)$.

Proof. Since any acyclic graphoidal cover is a graphoidal cover and any monophonic graphoidal cover is an acyclic graphoidal cover, we have $\eta(G) \leq$ $\eta_{a}(G) \leq \eta_{m}(G)$. Also, since every geodesic is a monophonic path, we have every 
geodesic graphoidal cover is a monophonic graphoidal cover and so $\eta_{m}(G) \leq$ $\eta_{g}(G)$. Hence $\eta(G) \leq \eta_{a}(G) \leq \eta_{m}(G) \leq \eta_{g}(G)$.

Remark 2.4. For the graph $K_{2}, \eta\left(K_{2}\right)=\eta_{a}\left(K_{2}\right)=1$, for the cycle $C_{5}$, $\eta_{a}\left(C_{5}\right)=\eta_{m}\left(C_{5}\right)=2$, for the cycle $C_{3}, \eta_{m}\left(C_{3}\right)=\eta_{g}\left(C_{3}\right)=3$. Further, for a tree $T, \eta(T)=\eta_{a}(T)=\eta_{m}(T)=\eta_{g}(T)=n-1$, where $n$ is the number of end vertices of $T$. All the inequalities in Theorem 2.3 can be strict. For the graph $G$ given in Figure 2.2, $\eta(G)=2, \eta_{a}(G)=3, \eta_{m}(G)=4$ and $\eta_{g}(G)=5$. Thus we have $\eta(G)<\eta_{a}(G)<\eta_{m}(G)<\eta_{g}(G)$.

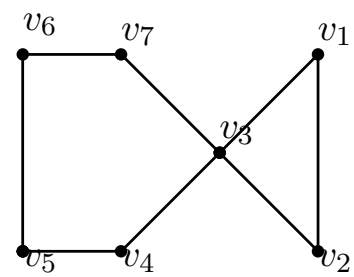

Figure 2.2: $G$

Since $q-p \leq \eta_{a}(G) \leq q$ and $\eta_{a}(G) \leq \eta_{m}(G) \leq q$, we have $q-p \leq \eta_{m}(G) \leq q$. Now, we proceed to characterize graphs $G$ for which the bounds of $\eta_{m}(G)$ are attained.

For any monophonic graphoidal cover $\psi_{m}$ of a graph $G$, let $t_{\psi_{m}}$ denote the number of vertices of $G$ which are not internal vertices of any path in $\psi_{m}$. Let $t_{m}=\min t_{\psi_{m}}$, where the minimum is taken over all graphoidal covers of $G$.

Theorem 2.5. For any graph $G, \eta_{m}(G)=q-p+t_{m}$.

Proof. Let $\psi_{m}$ be any monophonic graphoidal cover of $G$. Then $q=$ $\sum_{P \in \psi_{m}}|E(P)|=\left|\psi_{m}\right|+\sum_{P \in \psi_{m}} t_{m}(P)=\left|\psi_{m}\right|+p-t_{\psi_{m}}$. Therefore $\left|\psi_{m}\right|=$ $q-p+t_{\psi_{m}}$. Since $\eta_{m}(G)$ is the minimum cardinality of a monophonic graphoidal cover of $G$, we have $\eta_{m}(G)=q-p+t_{m}$.

Corollary 2.6. Let $T$ be a tree with $n$ pendant vertices. Then $\eta_{m}(T)=$ $n-1$.

Corollary 2.7. Let $G$ be a graph having $n$ simplicial vertices. Then $\eta_{m}(G) \geq q-p+n$. Furthermore, equality holds if and only if there exists a monophonic graphoidal cover $\psi_{m}$ of $G$ such that every non-simplicial vertex of $G$ is an internal vertex of a unique monophonic path in $\psi_{m}$.

The following proposition is the characterization result of the lower bound of $\eta_{m}(G)$ and it follows from Corollary 2.7. 
Proposition 2.8. For any connected graph $G$ of order at least $3, \eta_{m}(G)=$ $q-p$ if and only if $G$ has no simplicial vertices and there exists a monophonic graphoidal cover $\psi_{m}$ such that every vertex of $G$ is an internal vertex of a unique monophonic path in $\psi_{m}$.

Theorem 2.9. For any connected graph $G, \eta_{m}(G)=q$ if and only if $G$ is complete.

Proof. Let $G$ be a complete graph. Since any two vertices of $G$ are adjacent, the length of any monophonic path is one. Hence $E(G)$ is the unique monophonic graphoidal cover of $G$ and so $\eta_{m}(G)=q$.

Conversely, suppose that $\eta_{m}(G)=q$. Claim that $G$ is complete. If $G$ is not complete, then there exists a monophonic path, say $P$, in $G$ such that $|E(P)|>1$. Then $\psi_{m}=\{E(G)-E(P)\} \cup\{P\}$ is a monophonic graphoidal cover of $G$ and so $\eta_{m}(G) \leq q-1$, which is a contradiction.

Theorem 2.10. For any connected graph $G$ of order $p \geq 3$, $\eta_{m}(G)=q-1$ if and only if $G=K_{1}+\cup m_{j} K_{j}$, where $\sum m_{j} \geq 2$.

Proof. Let $\eta_{m}(G)=q-1$. Since $p \geq 3$, by Theorem 1.1 there exists a vertex $x$, which is not a cut vertex of $G$. If $G$ has two or more cut vertices, then let $P$ be a monophonic path containing at least two cut vertices. Then $|E(P)| \geq 3$. Clearly, $\psi_{m}=\{E(G)-E(P)\} \cup\{P\}$ is a monophonic graphoidal cover of $G$ and so $\eta_{m}(G) \leq\left|\psi_{m}\right|=q-2$, which is a contradiction. Thus the number of cut vertices $k$ of $G$ is at most one.

Case (i): If $k=0$, then the graph $G$ is a block. If $p=3$, then $G=K_{3}$ and so by Theorem 2.9, $\eta_{m}(G)=q$, which is a contradiction to the assumption. If $p \geq 4$, we claim that $G$ is complete. Suppose that $G$ is not complete. Then there exists two vertices $x$ and $y$ in $G$ such that $d(x, y) \geq 2$. By Theorem $1.2, x$ and $y$ lie on a common cycle and hence $x$ and $y$ lie on a smallest cycle $C=x, x_{1}, x_{2}, \ldots, y, \ldots, x_{n}, x$ of length at least 4 . Clearly, all the edges of $C$ lie on either an $x-y$ monophonic path, say $P_{1}$, or an $y-x$ monophonic path, say $P_{2}$. Then $\psi_{m}=\{E(G)-E(C)\} \cup\left\{P_{1}, P_{2}\right\}$ is a monophonic graphoidal cover of $G$ and so $\eta_{m}(G) \leq q-2$, which is a contradiction. Hence $G$ is complete and so by Theorem $2.9, \eta_{m}(G)=q$, which is again a contradiction. Thus $k \neq 0$.

Case (ii): If $k=1$, let $x$ be the cut vertex of $G$. If $p=3$, then $G=P_{3}=$ $K_{1}+\cup m_{j} K_{1}$ where $\sum m_{j}=2$. If $p \geq 4$, we claim that $G=K_{1}+\cup m_{j} K_{j}$, $\sum m_{j} \geq 2$. It is enough to prove that every block of $G$ is complete. Suppose that there exists a block $B$, which is not complete. Let $u$ and $v$ be two vertices in $B$ such that $d(u, v) \geq 2$. Then as in Case $(i), \eta_{m}(G) \leq q-2$, which is a 
contradiction. Thus every block of $G$ is complete so that $G=K_{1}+\cup m_{j} K_{j}$, where $K_{1}$ is the vertex $x$ and $\sum m_{j} \geq 2$.

Theorem 2.11. For any cycle $C_{p}(p \geq 4), \eta_{m}\left(C_{p}\right)=2$.

Proof. Let $C_{p}: v_{1}, v_{2}, v_{3}, \ldots, v_{p}, v_{1}$ be a cycle of order $p$. Then $\psi_{m}=$ $\left\{\left(v_{1}, v_{2}, v_{3}\right),\left(v_{3}, v_{4}, \ldots, v_{p}, v_{1}\right)\right\}$ is a minimum monophonic graphoidal cover of $C_{p}$ and hence $\eta_{m}\left(C_{p}\right)=2$.

Since every monophonic path in $K_{m, n}$ is a geodesic, we have the following result by Theorem 1.3.

Theorem 2.12. Let $K_{m, n}(1 \leq m \leq n)$ be a bipartite graph. Then

$$
\eta_{m}\left(K_{m, n}\right)= \begin{cases}1 & \text { if } m=1, n=1 \\ n-1 & \text { if } m=1, n \geq 2 \\ n & \text { if } m=2, n \geq 2 \\ m+n-1 & \text { if } m=3, n=3,4 \\ m+n & \text { if } m=3, n=5 \\ 2 n-3 & \text { if } m=3, n \geq 6 \\ m n-m-n & \text { if } m, n \geq 4 .\end{cases}
$$

Theorem 2.13. Let $G$ be a unicyclic graph with $n$ pendant vertices. Let $C$ be the unique cycle in $G$ having length greater than 3 and let $k$ be the number of vertices of degree greater than 2 on $C$. Then

$$
\eta_{m}(G)= \begin{cases}2 & \text { if } k=0 \\ n & \text { if there exists two non }- \text { adjacent vertices } \\ & \text { of degree }>2 \text { on } C \text { (or) all vertices in } C \text { are of } \\ & \text { degree }>2 \\ n+1 & \text { otherwise. }\end{cases}
$$

Proof. Let $C: v_{0}, v_{1}, v_{2}, \ldots, v_{l}, v_{0}$ be the unique cycle in $G$ having length greater than 3 .

Case $(i): k=0$. Then $G=C$ and by Theorem $2.11, \eta_{m}(G)=2$.

Case $(i i): k=1$. Let $v_{0}$ (say) be the unique vertex of degree greater than 2 on $C$. Let $G^{\prime}=G-\left\{v_{1}\right\}$. Then $G^{\prime}$ is a tree with $n+1$ pendant vertices and hence by Corollary 2.6, $\eta_{m}\left(G^{\prime}\right)=n$. Let $\psi_{m}^{\prime}$ be a minimum monophonic graphoidal cover of $G^{\prime}$. Clearly any path in $\psi_{m}^{\prime}$ is a monophonic path in $G$, we have $\psi_{m}=\psi_{m}^{\prime} \cup\left\{\left(v_{0}, v_{1}, v_{2}\right)\right\}$ is a monophonic graphoidal cover of $G$. Hence $\eta_{m}(G) \leq n+1$. 
Also, at least one vertex on $C$ and all the $n$ pendant vertices are exterior vertices of any minimum monophonic graphoidal cover of $G$, we have $t_{m} \geq n+1$. Then by Theorem 2.5, $\eta_{m}(G)=q-p+t_{m} \geq n+1$. Hence $\eta_{m}(G)=n+1$.

Case (iii): $k=2$ and the vertices of degree greater than 2 on $C$ are adjacent in $G$.

Let $v_{0}, v_{1}$ be vertices of degree greater than 2 on $C$. Let $P=\left(v_{1}, v_{2}, v_{3}\right)$ be a $v_{1}-v_{3}$ monophonic path in $G$. Let $G^{\prime}$ be the subgraph obtained by deleting $v_{2}$ from $G$. Clearly $G^{\prime}$ is a tree with $n+1$ pendant vertices and hence by Corollary 2.6, $\eta_{m}\left(G^{\prime}\right)=n$. If $\psi_{m}^{\prime}$ is a minimum monophonic graphoidal cover of $G^{\prime}$, then $\psi_{m}^{\prime} \cup\{P\}$ is a monophonic graphoidal cover of $G$ and hence $\eta_{m}(G) \leq n+1$. Also, at least one vertex on $C$ and all the $n$ pendant vertices are exterior vertices of any minimum monophonic graphoidal cover of $G$, we have $t_{m} \geq n+1$. Then by Theorem 2.5, $\eta_{m}(G) \geq n+1$. Hence $\eta_{m}(G)=n+1$.

Case (iv): $k \geq 2$ and there exists two non-adjacent vertices of degree greater than 2 on $C$.

Let $u, v$ be vertices of degree greater than 2 on $C$ such that all vertices in a $(u-v)$ - section of $C$ other than $u, v$ have degree 2 . Let $P$ denote this $(u, v)$ section and let $G^{\prime}$ be the subgraph obtained by deleting all the internal vertices of $P$. Clearly $G^{\prime}$ is a tree with $n$ pendant vertices and hence by Corollary 2.6, $\eta_{m}\left(G^{\prime}\right)=n-1$. If $\psi_{m}^{\prime}$ is a minimum monophonic graphoidal cover of $G^{\prime}$, then $\psi_{m}^{\prime} \cup\{P\}$ is a monophonic graphoidal cover of $G$ and hence $\eta_{m}(G) \leq n$. Also, since $G$ has $n$ pendant vertices, $t_{m} \geq n$ so that $\eta_{m}(G)=n$.

Case (v): $k \geq 3$ and all the vertices of $C$ are of degree greater than 2 .

Let $H=G-\left\{v_{1} v_{2}, v_{2} v_{3}\right\}$. Let $H^{\prime}$ and $H^{\prime \prime}$ be the components of $H$ with $H^{\prime}$ contain the vertices

$v_{1}, v_{3}$ and $H^{\prime \prime}$ contains the vertex $v_{2}$. Let $r$ be the number of pendant vertices in $H^{\prime}$ and let $s$ be the number of pendant vertices in $H^{\prime \prime}$. Since any pendant vertex of $H^{\prime}$ or $H^{\prime \prime}$ is a pendent vertex of $G$, we have $n=r+s$. Let $G^{\prime}=H^{\prime}$ and $G^{\prime \prime}=H^{\prime \prime} \cup\left\{v_{1} v_{2}, v_{2} v_{3}\right\}$. Then $G^{\prime}$ contains $r$ pendant vertices and $G^{\prime \prime}$ contains $s+2$ pendant vertices. Clearly $G^{\prime}$ and $G^{\prime \prime}$ are trees and hence by Corollary 2.6, $\eta_{m}\left(G^{\prime}\right)=r-1$ and $\eta_{m}\left(G^{\prime \prime}\right)=s+1$. Let $\psi_{m}^{\prime}$ be a minimum monophonic graphoidal cover of $G^{\prime}$ and let $\psi_{m}^{\prime \prime}$ be a minimum monophonic graphoidal cover of $G^{\prime \prime}$. Then $\psi_{m}^{\prime} \cup \psi_{m}^{\prime \prime}$ is a monophonic graphoidal cover of $G$ and hence $\eta_{m}(G) \leq r-1+s+1=n$. Also, since $G$ has $n$ pendant vertices, $t_{m} \geq n$ so that $\eta_{m}(G)=n$.

We have seen that if $G$ is a connected graph of order $p \geq 3$, then $q-p \leq$ $\eta_{m}(G) \leq q$. Also we have $\eta_{m}(G)=q-p$ if and only if $G$ has no simplicial vertices and there exists a monophonic graphoidal cover $\psi_{m}$ such that every 
vertex of $G$ is an internal vertex of a unique monophonic path in $\psi_{m}$ and $\eta_{m}(G)=q$ if and only if $G$ is complete. Also, it is proved that $\eta_{m}(G)=q-1$ if and only if $G=K_{1}+\cup m_{j} K_{j}$, where $\sum m_{j} \geq 2$. In the following theorem, we give an improved bounds for the monophonic graphoidal covering number of a graph interms of its size and monophonic diameter.

Theorem 2.14. For any connected graph $G$ of order $p \geq 2$, $\left\lceil q / d_{m}\right\rceil \leq \eta_{m}(G) \leq q-d_{m}+1$, where $d_{m}$ is the monophonic diameter of $G$.

Proof. Let $\psi_{m}$ be a minimum monophonic graphoidal cover of $G$. Since every edge of $G$ is in exactly one monophonic path in $\psi_{m}$, we have $q=$ $\sum_{P \in \psi_{m}}|E(P)|$. Since $|E(P)| \leq d_{m}$ for each $P$ in $\psi_{m}$, we have $q \leq \eta_{m}(G) . d_{m}$. Hence $\eta_{m}(G) \geq\left\lceil q / d_{m}\right\rceil$. Let $Q$ be a monophonic diametral path of $G$. It is clear that $\{(E(G)-E(Q)) \cup Q\}$ is a monophonic graphoidal cover of $G$. Hence $\eta_{m}(G) \leq|E(G)-E(Q)|+1=q-d_{m}+1$.

Now we give a realization result for the monophonic graphoidal covering number with some suitable conditions.

Theorem 2.15. For any positive integer $n$ with $q-p+2 \leq n \leq q-1$, there exists a tree $T$ such that the monophonic graphoidal covering number is n. .

Proof. Let $P: v_{1}, v_{2}, v_{3}, \ldots, v_{q-n+2}$ be a path of order $q-n+2$. Let $T$ be a tree obtained from $P$ by adding $n-1$ new vertices $u_{1}, u_{2}, \ldots u_{n-1}$ and joining each vertex $u_{i}(1 \leq i \leq n-1)$ to the vertex $v_{q-n+1}$. The tree $T$ is given in Figure 2.3 and it has $n+1$ pendant vertices. Then by Corollary 2.6, $\eta_{m}(T)=n$.

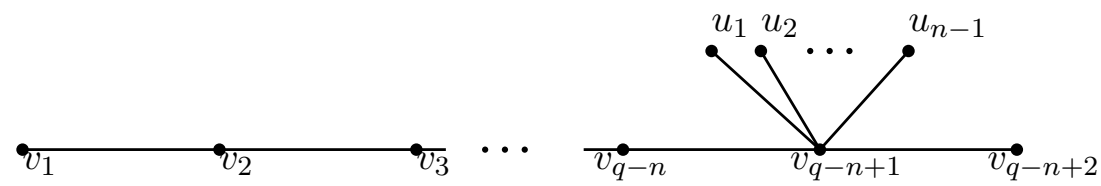

Figure 2.3: $T$

Remark 2.16. In a tree $T, q=p-1$ and so $q-p, q-p+1$ are non-positive numbers. Hence there does not exist a tree $T$ whose monophonic graphoidal covering number is either $q-p$ or $q-p+1$. Also, by Theorem $2.9, \eta_{m}(G)=q$ if and only if $G$ is complete. Thus there does not exist a tree with the monophonic graphoidal covering number is $q-p$ or $q-p+1$, or $q-1$. 
Problem 2.17. For any positive integer $n$ with $q-p \leq n \leq q$, does there exist a connected graph $G$ such that $G$ is not a tree and the monophonic graphoidal covering number is $n$ ?

\section{References}

[1] B. D. Acharya, Further results on the graphoidal covering number of a graph, Graph Theory News letter, 17 (4)(1988) 1.

[2] B. D. Acharya and E. Sampathkumar, Graphoidal covers and graphoidal covering number of a graph, Indian J. Pure Appl. Math., 18 (10) (1987) $882-890$.

[3] S. Arumugam and C. Pakkiam, Graphs with unique minimum graphoidal cover, Indian J. Pure Appl. Math., 25 (11) (1994) 1147 - 1153.

[4] S. Arumugam and J. Suresh Suseela, Acyclic graphoidal and path partitions in a graph, Discrete Mathematics, 190 (1998) 67 - 77.

[5] S. Arumugam and J. Suresh Suseela, Geodesic graphoidal covering number of a graph, J. Indian Math. Soc. New Ser. 72, No 1 - 4, 99 - 106 (2005).

[6] F. Harary, Graph Theory, Addison - Wesley, Reading Mass. (1969).

[7] C. Pakkiam and S. Arumugam, On the graphoidal covering number of a graph, Indian J. Pure Appl. Math., 20 (4) (1989) 330 - 333.

[8] C. Pakkiam and S. Arumugam, The graphoidal covering number of unicyclic graphs, Indian J. Pure Appl. Math., 23 (2) (1992) 141 - 143.

[9] A.P. Santhakumaran and P. Titus, Monophonic distance in graphs, Discrete Mathematics, Algorithms and Applications, Vol. 3, No. 2 (2011), 159169.

[10] A.P. Santhakumaran and P. Titus, A note on 'Monophonic Distance in Graphs', Discrete Mathematics, Algorithms and Applications, Vol. 4, No. 2 (2012), DOI: 10.1142/S1793830912500188. 
